\begin{tabular}{|c|c|}
\hline Title & $\begin{array}{l}\text { A new method to estimate pulmonary vascular resistance using diastolic pulmonary artery-right ventricular pressure } \\
\text { gradients derived from continuous wave Doppler velocity measurements of pulmonary regurgitation }\end{array}$ \\
\hline Author(s) & $\begin{array}{l}\text { Kaga, Sanae; Mikami, Tai sei; Muray ama, Michito; Okada, Kazunori; Masauzi, Nobuo; Nakabachi, Masahiro; Nishino, } \\
\text { Hisao; Y okoyama, Shinobu; Nishida, Mutsumi; Hay ashi, Taichi; Murai, Dai suke; Iwano, Hiroyuki; Sakakibara, } \\
\text { Mamoru; Y amada, Satoshi; T sutsui, Hiroyuki }\end{array}$ \\
\hline Citation & $\begin{array}{l}\text { International journal of cardiovascular imaging, 33(1), 31-38 } \\
\text { https://doi.org/10.1007/s10554-016-0965-2 }\end{array}$ \\
\hline Issue Date & 2017-01 \\
\hline Doc URL & http:/hdl.handle.net/2115/68252 \\
\hline Rights & $\begin{array}{l}\text { This is a post-peer-review, pre copyedit version of an article published in International journal of cardiovascular } \\
\text { imaging. The final authenticated version is available online at: http://dx.doi .org/10.1007/s10554-016-0965-2 }\end{array}$ \\
\hline Type & article (author version) \\
\hline File Information & Int J Cardiovasc Imaging_33(1)_31-38.pdf \\
\hline
\end{tabular}

Instructions for use 


\section{A New Method to Estimate Pulmonary Vascular Resistance Using Diastolic Pulmonary Artery-Right Ventricular Pressure Gradients Derived from Continuous-wave Doppler Velocity Measurements of Pulmonary Regurgitation}

Sanae Kaga, BS ${ }^{1,4}$, Taisei Mikami, MD, $\mathrm{PhD}^{1}$, Michito Murayama, $\mathrm{BS}^{2}$, Kazunori Okada, $\mathrm{PhD}^{1}$, Nobuo Masauzi, MD, $\mathrm{PhD}^{1}$, Masahiro Nakabachi, $\mathrm{MS}^{3}$, Hisao Nishino, $\mathrm{AS}^{3}$, Shinobu Yokoyama, $\mathrm{AS}^{3}$, Mutsumi Nishida, $\mathrm{PhD}^{3}$, Taichi Hayashi, $\mathrm{MD}, \mathrm{PhD}^{4}$, Daisuke Murai, $\mathrm{MD}, \mathrm{PhD}^{4}$, Hiroyuki Iwano, $\mathrm{MD}, \mathrm{PhD}^{4}$, Mamoru Sakakibara, $\mathrm{MD}, \mathrm{PhD}^{4}$, Satoshi Yamada, $\mathrm{MD}, \mathrm{PhD}^{4}$, Hiroyuki Tsutsui, $\mathrm{MD}, \mathrm{PhD}^{4}$

1. Faculty of Health Sciences, Hokkaido University, Kita-12, Nishi-5, Kita-ku, Sapporo 060-0812, Japan.

2. Graduate School of Health Sciences, Hokkaido University, Kita-12, Nishi-5, Kita-ku, Sapporo 060-0812, Japan.

3. Division of Laboratory and Transfusion Medicine, Hokkaido University Hospital, Kita-14, Nishi-5, Kita-ku, Sapporo 060-8648, Japan.

4. Department of Cardiovascular Medicine, Hokkaido University Graduate School of Medicine, Kita-15, Nishi-7, Kita-ku, Sapporo 060-8638, Japan.

Corresponding author: Dr. Taisei Mikami, Faculty of Health Sciences, Hokkaido University, Kita-12, Nishi-5, Kita-ku, Sapporo 060-0812, Japan. Tel.: +81-11-706-3403, Fax: +81-11-706-4916 E-mail address: mikami@hs.hokudai.ac.jp

Running title: Noninvasive Estimation of Pulmonary Vascular Resistance

Conflict of interest: None to declare 


\section{ABSTRACT}

Purpose: Pulmonary vascular resistance (PVR) is an important hemodynamic parameter in patients with heart failure, especially when the pulmonary arterial pressure is lower due to reduced stroke volume. Several echocardiographic methods to estimate PVR have been proposed, but their applications in patients with organic left-sided heart diseases have been limited. The aim of the present study was to examine the usefulness of our new method to estimate PVR (PVR $\left.{ }_{\mathrm{PR}}\right)$ based on the continuous-wave Doppler velocity measurements of pulmonary regurgitation in these patients.

Methods: In 43 patients who underwent right heart catheterization, $\mathrm{PVR}_{\mathrm{PR}}$ was calculated as the difference between the Doppler-derived early- and end-diastolic pulmonary artery (PA)-right ventricular (RV) pressure gradients divided by the cardiac output measured in the left ventricular outflow tract by echocardiography.

Results: The PVR ${ }_{\mathrm{PR}}$ correlated well with invasive PVR $\left(\mathrm{PVR}_{\mathrm{CATH}}\right)(\mathrm{r}=0.81, \mathrm{p}<0.001)$ without any fixed bias in Bland-Altman analysis. The conventional echocardiographic PVRs showed inadequate correlations with $\mathrm{PVR}_{\mathrm{CATH}}$, or a obvious overestimation of $\mathrm{PVR}_{\mathrm{CATH}}$. In the receiver operating characteristic analyses to determine the patients with abnormal elevation of $\mathrm{PVR}_{\mathrm{CATH}}(>3$ Wood units, WU), the area under the curve was the greatest for $\operatorname{PVR}_{\mathrm{PR}}(0.964)$ compared to the conventional PVRs (0.649-0.839). $\mathrm{PVR}_{\mathrm{PR}}$ had $83 \%$ sensitivity and $100 \%$ specificity at the optimal cut-off value of 3.10 WU in identifying patients with $\mathrm{PVR}_{\mathrm{CATH}}>3 \mathrm{WU}$.

Conclusion: Our simple and theoretical PVR $\mathrm{PR}_{\mathrm{P}}$ is useful for the noninvasive estimation of PVR. 
Kaga et al. Page 3

Keywords: pulmonary vascular resistance; echocardiography; pulmonary regurgitation; left heart failure 


\section{INTRODUCTION}

The evaluation of pulmonary artery (PA) pressure plays an important role in the diagnosis and follow-up of patients with various cardiopulmonary diseases [1]. However, PA pressure is blood flow-dependent, and decreases due to a reduction in cardiac output. In this setting, pulmonary vascular resistance (PVR) more accurately reflects the patient's condition than PA pressure. PVR is measured by right heart catheterization as the difference between the mean PA pressure and the PA wedge pressure divided by the cardiac output. However, the invasive nature of catheterization makes it difficult or impossible to perform daily or repetitive measurements of PVR. Although several noninvasive methods to estimate PVR based on echocardiographic measurements have been proposed [2-7], these methods used various empirical constants and/or ignored the left ventricular filling pressure, and thus their accuracy may be limited. The guidelines of the American Society of Echocardiography recommended not to use noninvasive estimations of PVR as a substitute for the invasive evaluation of PVR [8]. It has been reported that the early-diastolic and end-diastolic pressure gradients between the PA and the right ventricle (RV) derived from pulmonary regurgitation (PR) velocities measured by using continuous-wave Doppler echocardiography can reflect the mean PA pressure and the PA wedge pressure, respectively [7-10]. We therefore postulated that, by using these parameters, we could establish a simpler and more accurate noninvasive method to estimate PVR. This method uses the formula which is almost the same as the original one and includes the parameter reflecting left atrial pressure, and does not use any empirical constant. The aim of the present study was to examine the usefulness of our new method to estimate PVR based on the measurements of the 
early- and end-diastolic PA-RV pressure gradient by continuous-wave Doppler echocardiography in patients with left heart disease.

\section{METHODS}

\section{Patients}

The study subjects were 43 patients who underwent right heart catheterization and echocardiography under stable clinical condition within one day (range 2-30 hours, mean and SD 21 7 hours, median 22 hours) between January 2013 and December 2014 in the Department of Cardiovascular Medicine, Hokkaido University. Patients with atrial fibrillation and those with inadequate right heart catheterization pressure tracing were excluded. Patients with severe tricuspid regurgitation were also excluded because of potential inaccuracy of cardiac output measured by the thermodilution method. The study subjects consisted of 29 males and 14 females, and their ages ranged from 20 to 88 years (59 \pm 17 years old). They had ischemic cardiac disease in 20 , cardiomyopathy in 11 , valvular heart disease in 7 (3 with aortic stenosis, 2 after aortic valve replacement, 1 with mitral regurgitation and 1 after mitral valve repair), and other cardiovascular diseases in 5. Cardiac catheterization was performed for assessment of coronary artery stenosis in 23 , hemodynamic assessment of heart failure in 14 , diagnosis of structural heart disease in 5 and postoperative evaluation in 1 . Among our 43 patients, 13 had pulmonary hypertension (mean pulmonary artery pressure $\geq 25 \mathrm{mmHg}$ ) and were exclusively classified into the Group 2 (pulmonary hypertension due to left heart disease) according to the European Society of Cardiology and the European Respiratory Society (ESC/ERS) guidelines [1]. 
This study was approved by the Research Ethics Committee of Hokkaido University Hospital as a retrospective observational study.

\section{Right heart catheterization}

Right heart catheterization was performed using a water-filled catheter for hemodynamic measurements. The PA systolic pressure, PA diastolic pressure, mean PA pressure and PA wedge pressure were measured. Cardiac output was determined by the thermodilution method, and the PVR (Wood units, WU) was calculated using the following equation:

$$
\mathrm{PVR}_{\mathrm{CATH}}=(\text { mean PA pressure }-\mathrm{PA} \text { wedge pressure }) / \text { cardiac output }
$$

The abnormal elevation of PVR $\mathrm{PATH}_{\mathrm{C}}$ was defined as $>3 \mathrm{WU}$ according to the ESC/ERS guidelines [1].

\section{Echocardiographic examination}

Echocardiography was performed using an Aplio XG/Artida equipped with a 2.5/3.0 MHz probe (Toshiba Medical Systems, Otawara, Japan), an E9 with an M5S probe (GE Healthcare, Little Chalfont, Buckinghamshire, UK), or an iE 33with an S5 probe (Phillips, Einthoven, Netherlands). Basic echocardiographic parameters were measured according to the guidelines of the American Society of Echocardiography (ASE) [11]. Regional wall motion was scored using a standard 16-segment left ventricular model. Each segment was scored using a 4-point scale as follows: $1=$ normal, 2 = hypokinesis, 3 = akinesis, and 4 = dyskinesis. Tricuspid annular plane systolic excursion was measured in the apical four-chamber view. The peak early-diastolic transmitral flow velocity (E) 
and the early-diastolic mitral annular velocity at the interventricular septal side (septal e') were measured, and the ratio of E to e' (E/septal e') was calculated.

The flow velocity in the RV outflow tract was recorded by placing the sample volume at the level of the pulmonary valve annulus in the parasternal short-axis view, and the time velocity integral $\left(\mathrm{TVI}_{\mathrm{RVOT}}\right)$, acceleration time and ejection time were measured. The peak tricuspid regurgitant velocity (TRV) was measured as the highest of the velocities obtained from the lower parasternal and apical multiple views. RV isovolumic contraction time was obtained as the interval between the onset of tricuspid regurgitation and that of RV ejection flow, and the total systolic time was obtained as the sum of the isovolumic contraction time and ejection time [2].

The PR flow velocity was recorded using continuous-wave Doppler echocardiography. In order to minimize the incident angle of the beam to a PR jet, the color flow signal of PR jet was visualized in two mutually orthogonal planes from several different left parasternal echo windows, and the PR flow velocity was recorded from the echo window providing the least incident angle. The peak early-diastolic and end-diastolic velocities of PR were measured to calculate the early- and end-diastolic PA-RV pressure gradients using the simplified Bernoulli equation (Figure 1). The cardiac output was calculated from the time velocity interval of the left ventricular ejection flow and the diameter of the left ventricular outflow tract.

A contrast enhancement technique was not used in the continuous-wave Doppler recording. Every measurement was done in a cardiac cycle during a breath hold at shallow expiration or at the intermediate expiratory position under quiet respiration by a single observer (S.K.) blinded to the 
cardiac catheterization data.

We calculated the echocardiographic PVR (WU) reported by Scapellato et al. [2], that by Abbas et al. in 2003 [3], that by Dahiya et al. [4], that by Lindqvist et al. [5], that by Abbas et al. in 2013 [6], and that by our new method (PVR $\mathrm{PR})$ using the following equations:

PVR-Scapellato $=-0.156+1.154 \times[(\mathrm{RV}$ isovolumic contraction time $/$ acceleration time of RV ejection flow)/ total RV systolic time]

PVR-Abbas-2003 $=10 \times \mathrm{TRV} / \mathrm{TVI}_{\mathrm{RVOT}}+0.16$

PVR-Dahiya $=($ RV systolic pressure $-\mathrm{E} /$ septal e' $) / \mathrm{TVI}_{\mathrm{RVOT}}$

where RV systolic pressure $=4 \times \mathrm{TRV}^{2}+$ estimated right atrial pressure

PVR-Lindqvist $=0.95 \times[($ mean PA pressure -10$) /$ echocardiographic cardiac output $]-0.29$

where mean PA pressure $=\left(4 \times \mathrm{TRV}^{2}+10\right) \times 0.61+2$

PVR-Abbas-2013 $=5.19 \times \mathrm{TRV}^{2} / \mathrm{TVI}_{\mathrm{RVOT}}+0.4$

$\mathrm{PVR}_{\mathrm{PR}}=$ (early-diastolic PA-RV pressure gradient - end-diastolic PA-RV pressure gradient) / echocardiographic cardiac output

\section{Statistical Analyses}

Statistical analyses were performed using standard statistical software (SPSS II for Windows, SPSS,

Chicago, IL, USA). All numerical data were presented as a mean \pm SD. The relationship between variables was examined using a linear correlation and a regression analysis. A Bland-Altman analysis was done to assess a systematic error of echocardiography compared to right heart catheterization [12]. 
Wilcoxon rank sum test was used to compare continuous variables. A receiver operating characteristic analysis was used to evaluate the accuracy of echocardiographic PVR values for the discrimination of

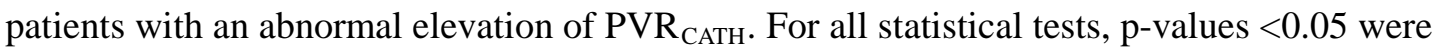
considered significant. Intraobserver and interobserver variabilities were assessed in 20 randomly selected subjects by one observer (S. K.) twice and by a second observer (M. M.).

\section{RESULTS}

The clinical, hemodynamic and basic echocardiographic characteristics of the studied patients are presented in Table 1. Out of the 43 study patients, we could measure PVR-Scapellato in 40 patients (93\%), PVR-Abbas-2003 in 36 patients (84\%), PVR-Dahiya in 35 patients (81\%), PVR-Lindqvist in 36 patients (84\%), PVR- Abbas-2013 in 36 patients (84\%) and $\mathrm{PVR}_{\mathrm{PR}}$ in 38 patients (88\%). TRV could be measured in 36 patients (84\%), peak early-diastolic PR velocity in 38 (88\%) and end-diastolic PR velocity in 39 (91\%).

Figure 2 shows the results of the correlation and regression analyses between the echocardiographic PVRs and PVR $\mathrm{CATH}_{\text {, }}$ and Figure 3 provides the results of Bland-Altman analyses. The correlation coefficient was $0.49(\mathrm{p}=0.001)$ in PVR-Scapellato, $0.54(\mathrm{p}=0.001)$ in PVR-Abbas-2003, 0.54 ( $\mathrm{p}=0.001)$ in PVR-Dahiya, 0.76 ( $\mathrm{p}<0.001)$ in PVR-Lindqvist, $0.66(\mathrm{p}<0.001)$ in PVR-Abbas-2013 and $0.81(\mathrm{p}<0.001)$ in $\mathrm{PVR}_{\mathrm{PR}}$. The correlation with $\mathrm{PVR}_{\mathrm{CATH}}$ was best for $\mathrm{PVR}_{\mathrm{PR}}$, followed by PVR-Lindqvist. 
fixed bias was revealed by the Bland-Altman analysis (mean 0.25; 95\% limits of mean agreement, -0.01 to 0.51$)$. On the other hand, PVR-Lindqvist was significantly greater than $\mathrm{PVR}_{\mathrm{CATH}}(3.58 \pm 3.06$ vs. 1.98 $\pm 1.17 \mathrm{WU}, \mathrm{p}<0.001$ ) and had a direct fixed bias (mean 1.61; 95\% limits of mean agreement, 0.85-2.36) showing distinct overestimation of PVR by this method.

In the receiver operating characteristic analyses to determine the patients with the abnormal elevation of $\mathrm{PVR}_{\mathrm{CATH}}(>3 \mathrm{WU})$, the area under the curve was the greatest for $\mathrm{PVR}_{\mathrm{PR}}(0.964)$ compared to PVR-Scapellato (0.697), PVR-Abbas-2003 (0.789), PVR-Dahiya (0.648), PVR-Lindqvist (0.839) and PVR-Abbas-2013 (0.789). PVR $_{\mathrm{PR}}$ had $83 \%$ sensitivity and $100 \%$ specificity at the optimal cut-off value of 3.10 WU in identifying patients with $\mathrm{PVR}_{\mathrm{CATH}}>3 \mathrm{WU}$ (Figure 4).

Intraobserver and interobserver measurements for $\mathrm{PVR}_{\mathrm{PR}}$ showed interclass correlation coefficients of 0.92 and 0.90 , respectively, indicating satisfactory reproducibility of the measurement of $\mathrm{PVR}_{\mathrm{PR}}$.

\section{DISCUSSION}

The results of the present study demonstrated that, in patients with the organic left-sided heart diseases, our new method using continuous-wave Doppler-derived PR velocities could more accurately estimate $\mathrm{PVR}_{\mathrm{CATH}}$ compared to the previous representative methods using echocardiography. Among the echocardiographic PVRs, $\mathrm{PVR}_{\mathrm{PR}}$ had the best correlation and agreement with $\mathrm{PVR}_{\mathrm{CATH}}$. Moreover, $\mathrm{PVR}_{\mathrm{PR}}$ could identify patients with abnormally elevated PVR $_{\text {CATH }}$ with $83 \%$ sensitivity and $100 \%$ specificity at the optimal cut-off value $3.10 \mathrm{WU}$, which is 
nearly equal to the catheter cut-off value of 3 WU defined in the ESC/ERS guidelines. Our method is simple; it requires measurements of only the PR velocities and cardiac output, and is consistent with the original formula being used for the invasive measurement without using any empirical constant. Moreover, by including the variable reflecting left atrial pressure, this method enabled the assessment of PVR in patients with post-capillary pulmonary hypertension associated with left-sided heart diseases, in whom the accurate assessment of PVR had been difficult by previous noninvasive methods.

Several noninvasive methods to estimate PVR based on echocardiographic measurements have been proposed previously (Table 2). However, the guidelines of the ASE recommended not to use noninvasive estimations of PVR as a substitute for the invasive evaluation of PVR [8]. In the present study, PVR-Scapellato, PVR-Abbas-2003 and PVR-Abbas-2013 inadequately correlated with PVR $_{\text {CATH }}$ in our patients with post-capillary pulmonary hypertension, probably due to the lack of any parameter reflecting left atrial pressure in their equation. The correlation between PVR-Dahiya and PVR $_{\text {CATH }}$ was not satisfactory, probably because the ability of E/e' to estimate PA wedge pressure was not very high $[13,14]$. PVR-Lindqvist showed a good correlation with $\mathrm{PVR}_{\mathrm{CATH}}(\mathrm{r}=0.76)$, comparable to our method ( $\mathrm{r}=0.81)$. However, their method distinctly overestimated PVR $\mathrm{CATH}_{\mathrm{H}}$, and this method may not be applicable to patients with post-capillary pulmonary hypertension.

It has been reported that PR velocity could be recorded by continuous-wave Doppler in 74\%-86 \% of patients in a clinical setting [15]. In our series, both the early-diastolic and end-diastolic PR velocities, and thus the $\mathrm{PVR}_{\mathrm{PR}}$, could be obtained in 38 of 43 patients (88\%). The measurable rate 
of our method was not very high, but was similar to those of the other conventional methods except for the Scapellato method. It has been reported that the early-diastolic pressure gradient derived from the peak early-diastolic PR velocity is useful for estimating the mean PA pressure [7-10], whereas the end-diastolic pressure gradient calculated from the end-diastolic PR velocity reflects end-diastolic PA pressure $[7,9]$. It was also reported that the end-diastolic PA pressure measured by Doppler echocardiography correlated well with invasively measured PA wedge pressure and might be used as its surrogate [10]. Thus, in our new method, $\mathrm{PVR}_{\mathrm{PR}}$ can be calculated using a formula that is very similar to that used for the $\mathrm{PVR}_{\mathrm{CATH}}$, without any empirical constant. Our method requires the measurements of only two velocities from a single PR velocity recording and the cardiac output in the left ventricular outflow tract, and it may be one of the simplest methods to measure PVR among the noninvasive methods reported previously.

The systolic RV-right atrial pressure gradient can be noninvasively obtained using TRV [8], and it can be used to estimate the mean PA pressure as in the first and second Abbas [3, 6], Dahiya [4], and Lindqvist methods [5]. However, a wide regurgitant orifice may develop most frequently in the tricuspid valve among the four cardiac valves, probably because of the easy distensibility of right heart structures around the tricuspid annulus [16, 17]. A very wide regurgitant orifice leads to laminar regurgitant flow instead of turbulent flow [18, 19], and the peak systolic RV-right atrial pressure gradient can be greatly overestimated by the simplified Bernoulli equation due to the pressure recovery phenomenon. Such a severe functional tricuspid regurgitation may frequently develop in patients with long-standing pulmonary hypertension. Such a situation can cause a decrease in PA 
pressure despite the deteriorated hemodynamics due to low flow, and it typically requires the assessment of PVR instead of PA pressure. Although an overestimation of pressure gradients can also occur in patients with severe PR, such patients may be rare and limited to those after surgery for tetralogy of Fallot. Thus, our method is considered to be especially useful to evaluate patients with both left and right heart failure.

There are several limitations in this study. First, right heart catheterization and echocardiography were not performed simultaneously, and thus the hemodynamic alternations might not be completely excluded due to the study's retrospective nature. Second, the sample size of the present study was fairly small. Third, some skill may be required for examiners to accurately measure both the earlyand end-diastolic PR velocities to estimate PVR using our method. Finally, although the PA diastolic pressure is known to correspond well with the mean PA wedge pressure in patients with left heart failure, it is also reported that the PA diastolic pressure might be significantly higher than the PA wedge pressure in patients with pre-capillary hypertension such as pulmonary arterial hypertension, pulmonary thromboembolism, lung disease and tachycardia [20]. Thus, our method may not be applicable to patients with these conditions.

\section{CONCLUSION}

Our new method based on the continuous-wave Doppler measurements of early- and end-diastolic PA-RV pressure gradients is useful for the noninvasive estimation of PVR in patients with left-sided organic heart diseases. 


\section{REFERENCES}

1. Galiè N, Humbert M, Vachiery JL, Gibbs S, Lang I, Torbicki A, Simonneau G, Peacock A, Vonk Noordegraaf A, Beghetti M, Ghofrani A, Gomez Sanchez MA, Hansmann G, Klepetko W, Lancellotti P, Matucci M, McDonagh T, Pierard LA, Trindade PT, Zompatori M, Hoeper M, Aboyans V, Vaz Carneiro A, Achenbach S, Agewall S, Allanore Y, Asteggiano R, Paolo Badano L, Albert Barberà J, Bouvaist H, Bueno H, Byrne RA, Carerj S, Castro G, Erol Ç, Falk V, Funck-Brentano C, Gorenflo M, Granton J, Iung B, Kiely DG, Kirchhof P, Kjellstrom B, Landmesser U, Lekakis J, Lionis C, Lip GY, Orfanos SE, Park MH, Piepoli MF, Ponikowski P, Revel MP, Rigau D, Rosenkranz S, Völler H, Luis Zamorano J. 2015 ESC/ERS Guidelines for the diagnosis and treatment of pulmonary hypertension: The Joint Task Force for the Diagnosis and Treatment of Pulmonary Hypertension of the European Society of Cardiology (ESC) and the European Respiratory Society (ERS): Endorsed by: Association for European Paediatric and Congenital Cardiology (AEPC), International Society for Heart and Lung Transplantation (ISHLT). Eur Heart 2016; 37: 67-119.

2. Scapellato F, Temporelli PL, Eleuteri E, Corrà U, Imparato A, Gianuzzi P. Accurate noninvasive estimation of pulmonary vascular resistance by Doppler echocardiography in patients with chronic heart failure. J Am Coll Cardiol 2001; 37: 1813-1819.

3. Abbas AE, Fortuin FD, Schiller NB, Appleton CP, Moreno CA, Lester SJ. A simple method for noninvasive estimation of pulmonary vascular resistance. J Am Coll Cardiol 2003; 41:

1021-1027. 
4. Dahiya A, Vollbon W, Jellis C, Prior D, Wahi S, Marwick T. Echocardiographic assessment of raised pulmonary vascular resistance: application to diagnosis and follow-up of pulmonary hypertension. Heart 2010; 96: 2005-2009.

5. Lindqvist P, Söderberg S, Gonzalez MC, Tossavainen E, Henein MY. Echocardiography based estimation of pulmonary vascular resistance in patients with pulmonary hypertension: a simultaneous Doppler echocardiography and cardiac catheterization study. Eur J Echocardiogr 2011; 12: 961-966.

6. Abbas AE, Franey LM, Marwick T, Maeder MT, Kaye DM, Vlahos AP, Serra W, Al-Azizi K, Schiller NB, Lester SJ. Noninvasive assessment of pulmonary vascular resistance by Doppler echocardiography. J Am Soc Echocardiogr 2013; 26: 1170-1177.

7. Milan A, Magnino C, Veglio F. Echocardiographic indexes for the non-invasive evaluation of pulmonary hemodynamics. J Am Soc Echocardiogr 2010; 23: 225-239.

8. Rudski LG, Lai WW, Afilalo J, Hua L, Handschumacher MD, Chandrasekaran K, Solomon SD, Louie EK, Schiller NB. Guidelines for the echocardiographic assessment of the right heart in adults: a report from the American Society of Echocardiography endorsed by the European Association of Echocardiography, a registered branch of the European Society of Cardiology, and the Canadian Society of Echocardiography. J Am Soc Echocardiogr 2010; 23: 685-713.

9. Nagueh SF, Appleton CP, Gillebert TC, Marino PN, Oh JK, Smiseth OA, Waggoner AD, Flachskampf FA, Pellikka PA, Evangelista A. Recommendations for the evaluation of left ventricular diastolic function by echocardiography. J Am Soc Echocardiogr 2009; 22: 107-133. 
10. Lee RT, Lord CP, Plappert T, Sutton MS. Prospective Doppler echocardiographic evaluation of pulmonary artery diastolic pressure in the medical intensive care unit. Am J Cardiol 1989; 64: 1366- 1370.

11. Lang RM, Badano LP, Mor-Avi V, Afilalo J, Armstrong A, Ernande L, Flachskampf FA, Foster E, Goldstein SA, Kuznetsova T, Lancellotti P, Muraru D, Picard MH, Rietzschel ER, Rudski L, Spencer KT, Tsang W, Voigt JU. Recommendations for cardiac chamber quantification by echocardiography in adults: an update from the American Society of Echocardiography and the European Association of Cardiovascular Imaging. J Am Soc Echocardiogr 2015; 28: 1-39.

12. Bland JM, Altman DG. Statistical methods for assessing agreement between two methods of clinical measurement. Lancet 1986; 1: 307-310.

13. Geske JB, Sorajja P, Nishimura RA, Ommen SR. Evaluation of left ventricular filling pressures by Doppler echocardiography in patients with hypertrophic cardiomyopathy: correlation with direct left atrial pressure measurement at cardiac catheterization. Circulation 2007; 116: 2702-2708.

14. Mullens W, Borowski AG, Curtin RJ, Thomas JD, Tang WH. Tissue Doppler imaging in the estimation of intracardiac filling pressure in decompensated patients with advanced systolic heart failure. Circulation 2009; 119: 62-70.

15. Borgeson DD, Seward JB, Miller FA Jr, Oh JK, Tajik AJ. Frequency of Doppler measurable pulmonary artery pressures. J Am Soc Echocardiogr 1996; 9: 832-837.

16. Tei C, Pilgrim JP, Shah PM, Ormiston JA, Wong M. The tricuspid valve annulus: study of size 
and motion in normal subjects and in patients with tricuspid regurgitation. Circulation 1982; 66:

$665-671$.

17. Mikami T, Kudo T, Sakurai N, Sakamoto S, Tanabe Y, Yasuda H. Mechanisms for development of functional tricuspid regurgitation determined by pulsed Doppler and two-dimensional echocardiography. Am J Cardiol 1984; 53: 160-163.

18. Minagoe S, Rahimtoola SH, Chandraratna PA. Significance of laminar systolic regurgitant flow in patients with tricuspid regurgitation: a combined pulsed-wave, continuous-wave Doppler and two-dimensional echocardiographic study. Am Heart J 1990; 119: 627-635.

19. Cobey FC, Fritock M, Lombard FW, Glower DD, Swaminathan M. Severe tricuspid valve regurgitation: a case for laminar flow. J Cardiothorac Vasc Anesth 2012; 26: 522-524.

20. Davidson CJ, Bonow RO. Cardiac Catheterization. In: Bonow RO, Mann DL, Zipes DP, Libby P, ed. Braunwald’s Heart Disease, 9th ed. Philadelphia: Elsevier Saunders, 2012: 383-405. 


\section{FIGURE LEGENDS}

Figure 1. Measurement of pulmonary regurgitant velocities.

A continuous-wave Doppler recording of pulmonary regurgitation is shown. The peak early-diastolic velocity (V1) and end-diastole velocity (V2) were measured to calculate the early- and end-diastolic pulmonary-right ventricle pressure gradient, respectively.

Figure 2. Correlation and regression analyses between the pulmonary vascular resistance (PVR) obtained using catheterization (PVR ${ }_{\mathrm{CATH}}$ ) and the PVRs estimated using echocardiography.

(A) Echocardiographic PVR reported by Scapellato et al., (B) Abbas et al. in 2003, (C) Dahiya et al., (D) Lindqvist et al., (E) Abbas et al. in 2013, and (F) by our method (PVR $\left.{ }_{\mathrm{PR}}\right)$.

Figure 3. Bland-Altman analyses between the pulmonary vascular resistance (PVR) obtained

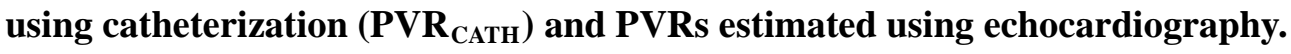

(A) Echocardiographic PVR reported by Scapellato et al., (B) Abbas et al. in 2003, (C) Dahiya et al., (D) Lindqvist et al., (E) Abbas et al. in 2013, and (F) our method (PVR $\left.{ }_{\mathrm{PR}}\right)$.

Figure 4. Receiver operating characteristic curves to discriminate patients with pulmonary vascular resistance $(\mathrm{PVR})$ obtained using catheterization $\left(\mathrm{PVR}_{\mathrm{CATH}}\right)>3 \mathrm{WU}$ by the echocardigraphic PVRs. 
(A) Echocardiographic PVR reported by Scapellato et al., (B) Abbas et al. in 2003, (C) Dahiya et al.,

(D) Lindqvist et al., (E) Abbas et al. in 2013, and (F) our method ( $\left.P R_{P R}\right)$. Auc was the greatest

(0.964) for PVR $\mathrm{PR}_{\mathrm{P}}$ by our method compared to other previous methods (0.649-0.839).

AUC, area under the curve. 
Table 1. Patient characteristics

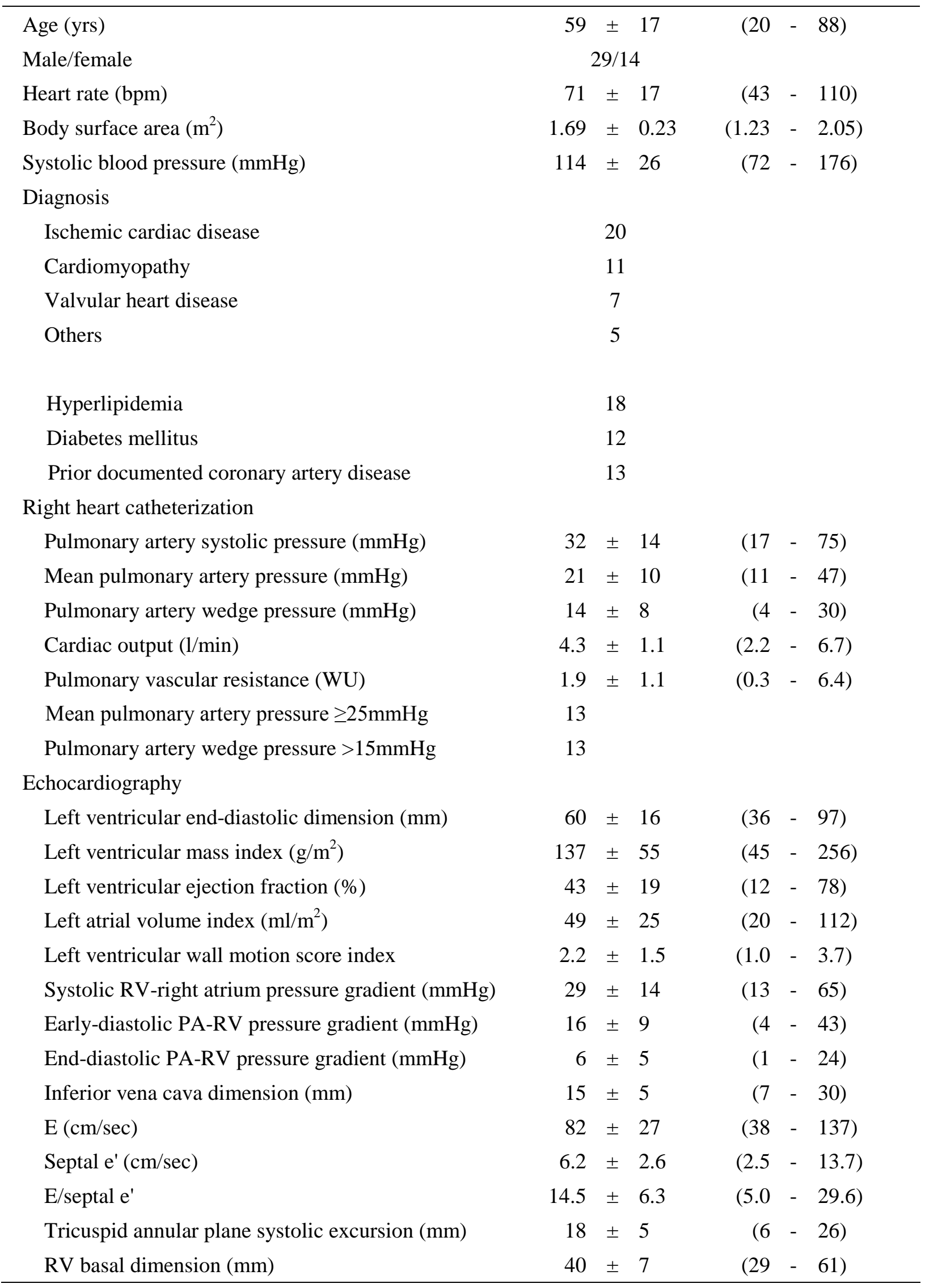

Data are mean \pm SD or number of patients. 
RV, right ventricle; PA, pulmonary artery; E, early diastolic transmitral flow velocity; e', early diastolic mitral annular velocity. 
Figure 1

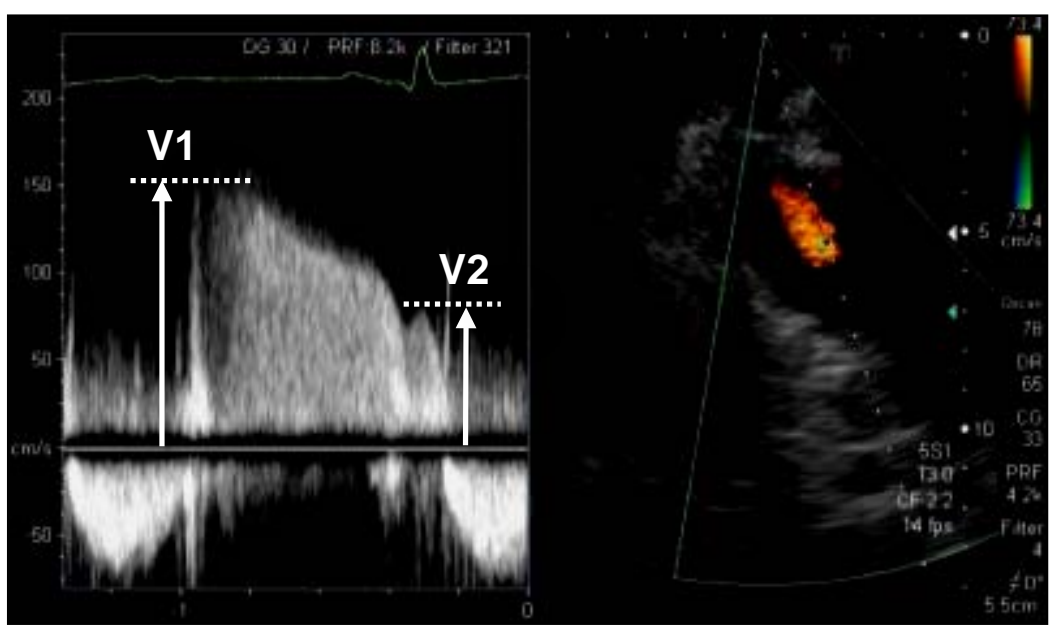


Figure 3

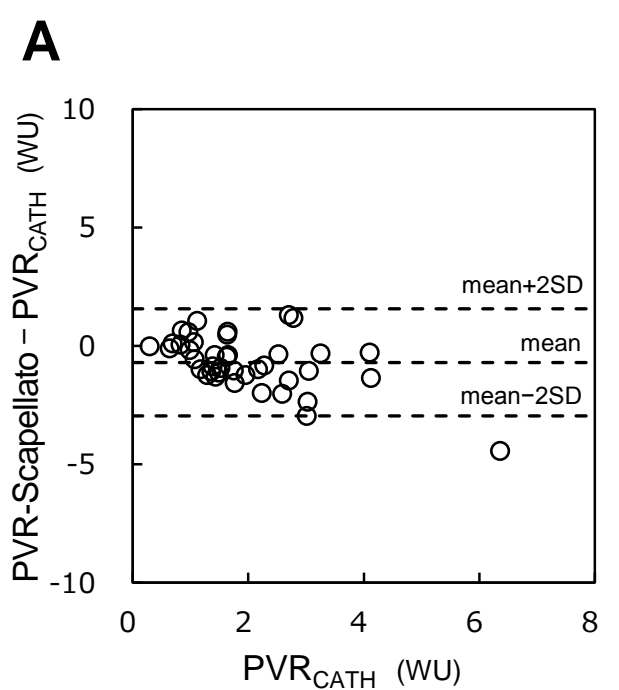

D

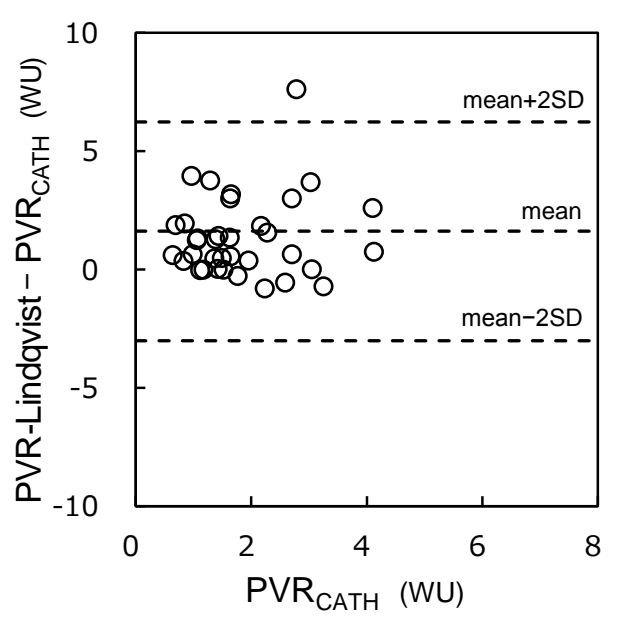

B

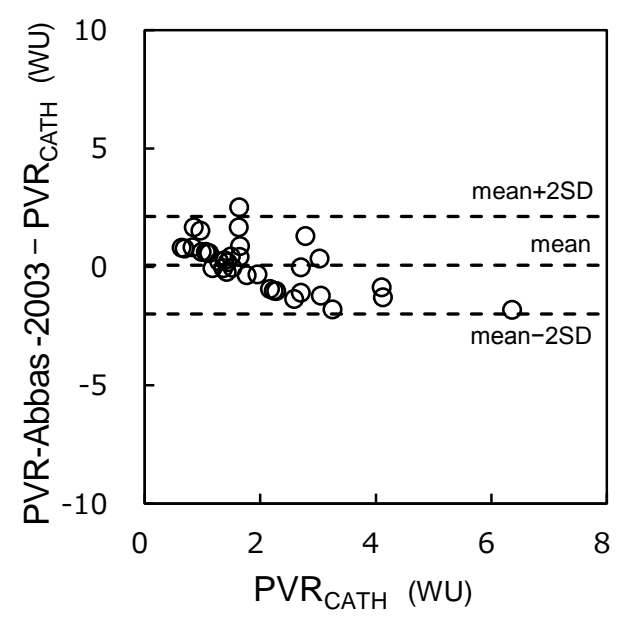

E

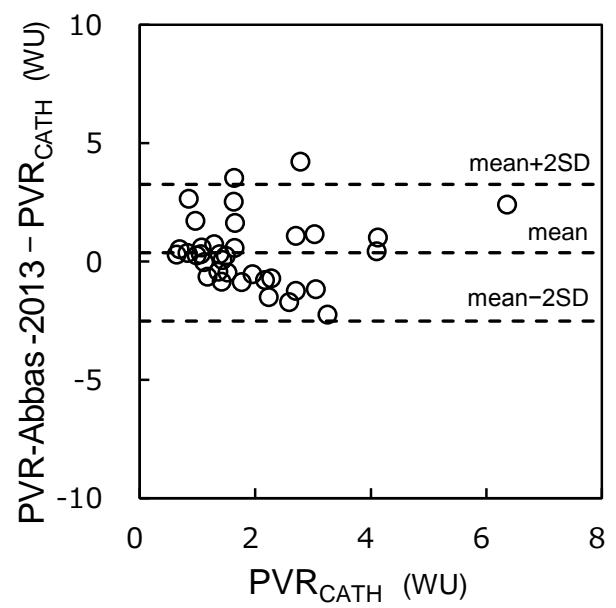

C

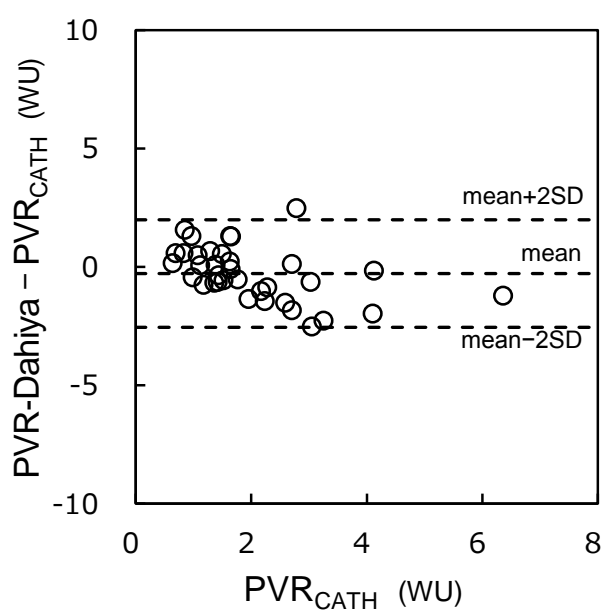

F

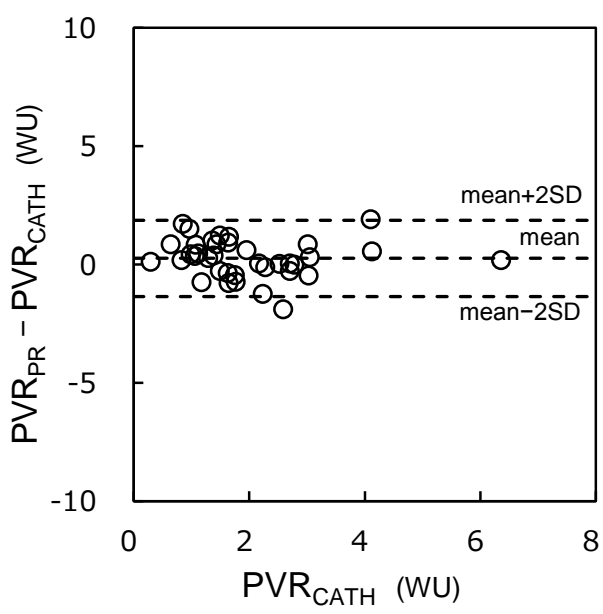




\section{Figure 4}
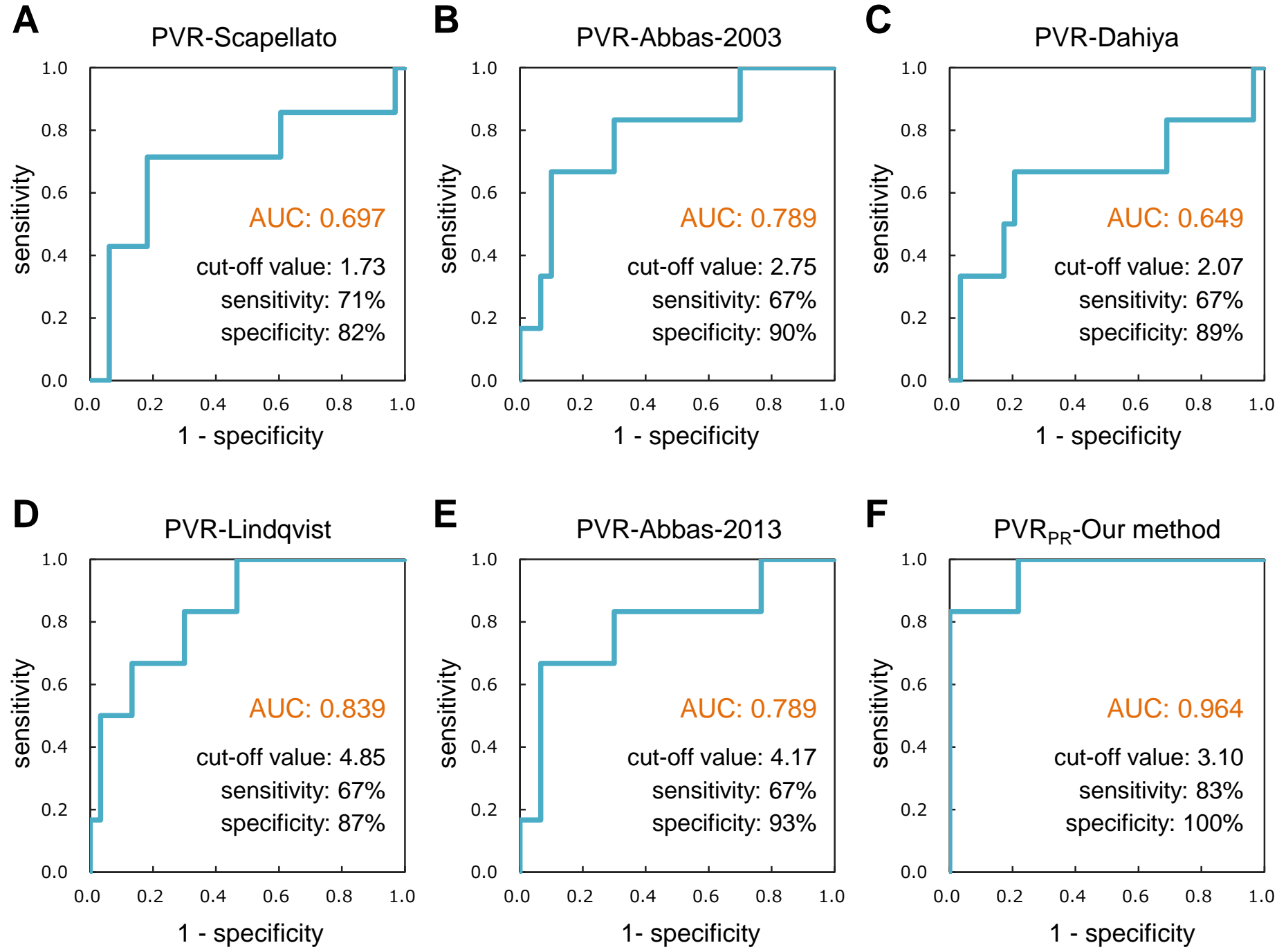